\title{
Development of EEG based Emotion Recognition System using Song Induced Activity
}

\author{
Rakesh S Deore \\ H.O.D \\ Dept. of Computer Science \\ S.S.V.P.S's Science College, \\ Dhule (M.S), India
}

\author{
Rahul D Chaudhari \\ Lecturer \\ Dept. of Computer Science \\ S.S.V.P.S`s Science College, \\ Dhule (M.S), India
}

\author{
Suresh C Mehrotra \\ Professor \\ Dept of Computer Science \\ BAMU \\ Aurangabad
}

\begin{abstract}
In this study we build a mood recognition system using EEG signal of Song Induced activity. In this we have analyzed alpha EEG powers related to left hemisphere, right hemisphere regions of brains. This has given the significance of different brain region related to emotions. This study successfully achieves the goal to design a system which offers offline mood recognition system. In this study we show that it is possible to recognize the different moods of person using EEG signal. We observe the different brain locations as Left Hemisphere and Right Hemisphere to recognize the significance according to different moods. The alpha powers are more alert during National, Happy, Romantic mood as compared to Sad mood. So it is possible to distinguish these different moods using alpha power values. The distance matrices also shows that it is possible to differentiate the emotions of persons using alpha power values.
\end{abstract}

\section{Keywords}

Human Computer Interface, EEG, Brain Regions.

\section{INTRODUCTION}

Music is often referred to as language of emotions. The sub cortical emotion processing parts of the brain affect the rest if body through two basic mechanisms: the release of chemical molecules into blood that act on various parts of body and the spread of neural activation to various brain centers and muscles. Through these mechanisms, the experience of an emotion is connected with a myriad of physiological responses, from muscle contractions, to change in breathing and heart rate, to changes in blood flow in various parts of the body, to sweating. Music produce autonomic changes associated with emotional processing.

\section{BACKGROUND AND MOTIVATION}

Number of different researchers work to associate the music and EEG signal to develop mood recognition system. Ito, S.I.; Mitsukura, Y.; Fukumi, M.; Akamatsu, N. (2003) processed the EEG pattern in 4 conditions, which are listening to Rock music, Schmaltzy Japanese ballad music, Healing music, and Classical music. They proposed the EEG analysis method by using the FA and the NN[1]. In 2005 Huisheng Lu; Mingshi Wang; Hongqiang Yu proposed a method whose aim is to study and confirm the character of EEG and the location in brain when a person was enjoying different rhythm music. It made the subjects excited when they enjoyed different rhythm music, the EEG signals are collected with Phoenix Digital EEG with 128 channels, and compared with the ones before the subjects enjoying the music. Obvious differences have been found between them. And the character of EEG has a little differences when the subjects enjoyed different rhythm music [2].In the year 2006 Karthick, N.G.; Thajudin, A.V.I.; Joseph, P.K. studied two types of music on the electroencephalogram (EEG) activity. The scaling properties of the EEG are studied using the detrended fluctuation analysis (DFA) algorithm, and the complexity of the electroencephalogram signal is quantified by the multiscale entropy (MSE) method. It was found that both methods show significant difference in the values of the estimated parameters for the electroencephalogram with and without music.[3]. Ito, S.-i.; Mitsukura, Y.; Fukumi, M.; Jianting Cao proposed a method for detecting the mood much music for prefrontal cortex electroencephalogram (EEG) activity[4]. Yuan-Pin Lin; Chi-Hong Wang; Tien-Lin Wu; Shyh-Kang Jeng; Jyh-Horng Chen gives an approach to recognize the emotion responses during multimedia presentation using the electroencephalogram (EEG) signals[5]. Vijayalakshmi, K.; Sridhar, S.; Khanwani, P.analysed the acute central system effects of relaxation techniques[6]. Yuan-Pin Lin; Chi-Hong Wang; Tzyy-Ping Jung; Tien-Lin Wu; Shyh-Kang Jeng; JengRen Duann; Jyh-Horng Chen gives a practical system for noninvasive assessment of the emotional states in practical or clinical applications.

\section{EXPERIMENTAL SETUP \\ 3.1 Methodology}

Music induces emotion in mind. These emotions are dependent not only types of music, but also sensitivity of the person subjected to music. However, songs may be classified as per their general effect on mind. For our study these classification has been done on the basis of song's appeal with respect to patriotism, happiness, romantic or sadness. Understanding of induced brain signals due to hearing of music will be essential information for training computers to identify different types of music.

\subsection{Subject Selection}

EEG recordings of 10 male right-handed subjects in the age group of (20-25) were taken. The subjects were normal without any mental disorder. They did not have any problem in communicating and had normal vision. Subjects were made to sit comfortably on an arm chair facing the screen in electromagnetically shielded room. The subjects had given their written consent for recording EEG signals before participating.

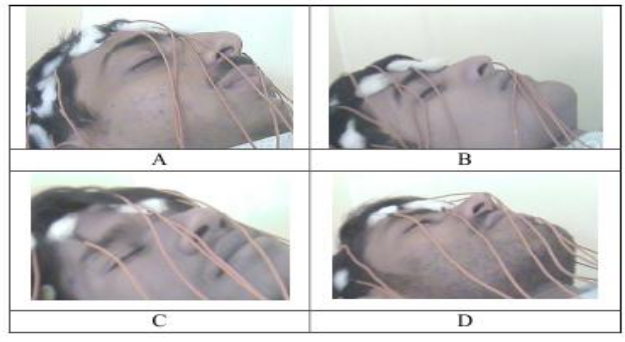

Fig 1.1 During Acquisition of EEG signals 


\subsection{Procedure:}

All subjects were instructed that this experiment has been designed to be used for HCI as mood recognition system. They came to the laboratory and were instructed about the nature of task which was to be administered. In this study, we have designed EEG dataset containing data of five mental tasks of ten different subjects. Subject sleep was conducted on a normal bed, relaxed arms resting on their legs. The electrodes were placed on scalp of the subject as per the International 10-20 standard. The test was conducted for 25 minutes, with eye closed and each subject was asked to perform these tasks. For all modes, the subjects were asked to lie on the bed along with the head phones.

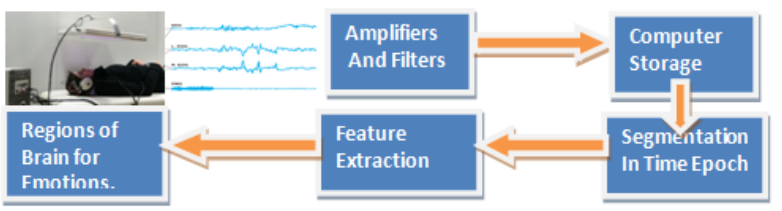

Fig 1.2 Overview of offline BCI system for mood recognition

\subsection{Apparatus and Recording procedure:}

The EEG signals, for each mode, was captured by RMS (Recorders \& Medicare Systems) EEG-32 Super Spac machine shown in figure 4.10 , for 5 minutes with sampling rate of $250 / \mathrm{s}$. The other parameters of the EEG machine were set as follows: low filter: $1 \mathrm{~Hz}$, high filter: $70 \mathrm{~Hz}$, sensitivity: $7 \mu \mathrm{V}$, number of channels: 17 , sweep speed: $30 \mathrm{~mm} / \mathrm{s}$ and Montage: BP PARA (R) .

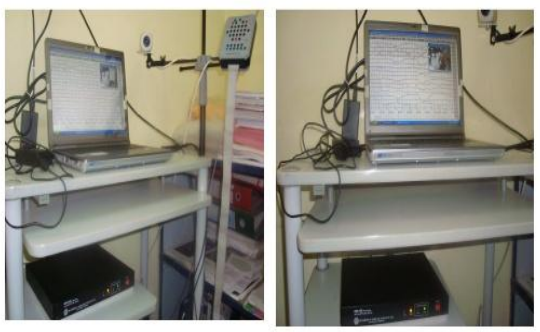

Fig 1.3 Photograph of RMS-EEG -32 Super Spec Machine

The experiment was conducted on 10 subjects . The electrodes were placed on scalp of subjects as per the international 10-20 standard. As given in Fig. 1.4

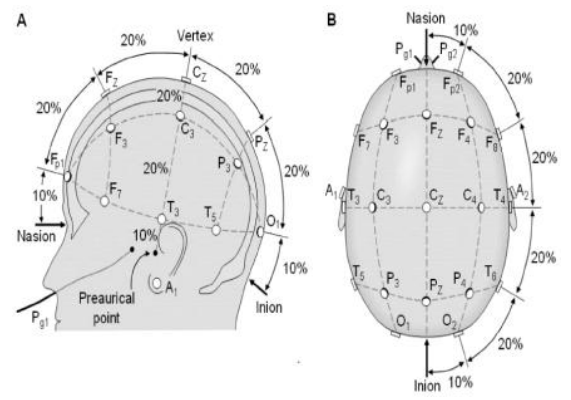

Fig 1.4 International 10-20 system for placement of electrode
The recording was captured for 5 minutes. This process was repeated for number of task times. The different parameters of the EEG machine were set as follows: low filter $1 \mathrm{~Hz}$, High filter $70 \mathrm{~Hz}$, sensitivity at $7 \mu \mathrm{V}$, number of channel 19 , sweep speed $30 \mathrm{~mm} / \mathrm{s}$, Montage set BPPARA (R) for all the experiments.

\section{ANALYSIS OF EEG DATA}

The following tables gives an example of data in relaxation mood, Happy Mood, Sad Mood , National Mood and Romantic mood. The table shows the data of all four frequency power band but we are mainly interested in alpha power. Here we also calculate the mean alpha power frequency. Here data is organized as left hemisphere, right hemisphere and center hemisphere.

Table 1.1 Alpha Power EEG data of all subjects in Left Hemisphere

\begin{tabular}{|c|c|c|c|c|c|}
\hline Subject & Relax & Happy & Sad & National & Romantic \\
\hline Subject1 & 4.763931 & 2.960641 & 3.822434 & 2.819911 & 3.156809 \\
\hline Subject2 & 12.34842 & 9.966793 & 10.53576 & 11.89535 & 9.743734 \\
\hline Subject3 & 1.816595 & 1.068355 & 1.499737 & 1.556776 & 2.154868 \\
\hline Subject4 & 15.18015 & 20.16189 & 19.44457 & 17.87863 & 20.13546 \\
\hline Subject5 & 7.822977 & 3.698947 & 4.228569 & 4.247039 & 2.867928 \\
\hline Subject6 & 0.419326 & 0.531201 & 0.616135 & 0.409704 & 0.544227 \\
\hline Subject7 & 4.300641 & 1.120938 & 2.64528 & 0.947023 & 1.488964 \\
\hline Subject8 & 1.689359 & 0.859984 & 0.713832 & 0.863931 & 0.739342 \\
\hline Subject9 & 2.255822 & 1.648109 & 2.036316 & 1.590066 & 1.84273 \\
\hline Subject10 & 2.995 & 0.990461 & 1.679556 & 0.905477 & 1.114153 \\
\hline Average & $\mathbf{5 . 3 5 9 2 2 2}$ & $\mathbf{4 . 3 0 0 7 3 2}$ & $\mathbf{4 . 7 2 2 2 1 9}$ & $\mathbf{4 . 3 1 1 3 9 1}$ & $\mathbf{4 . 3 7 8 8 2 2}$ \\
\hline
\end{tabular}

Table 1.1 shows the mean values of alpha power of different electrodes of all subjects in left hemisphere region. The table contains the mean values of alpha power of different relax mode, Happy Mode, Sad Mode, National Mode and Romantic mode. We also calculate the average values for all subjects. From table it is clear that the alpha value for relax mode is more this means that less activity in brain for relax mode. It is also observed that we are less alert in sad mode as compared to Happy, National and Romantic mode. So it is possible to make clear distinction between the different activities in left hemisphere.

Table 1.2 Distance Matrix of Alpha Power EEG data of all subject in Left Hemisphere

\begin{tabular}{|c|c|c|c|c|c|}
\hline & Relax & Happy & Sad & National & Romantic \\
\hline Relax & 0 & 1.0584 & 0.6370 & 1.0478 & 0.9804 \\
\hline Happy & 1.0584 & 0 & 0.4214 & 0.0106 & 0.0780 \\
\hline Sad & 0.6370 & 0.4214 & 0 & 0.4108 & 0.3433 \\
\hline National & 1.0478 & 0.0106 & 0.4108 & 0 & 0.06743 \\
\hline Romantic & 0.9804 & 0.0780 & 0.3433 & 0.06743 & 0 \\
\hline
\end{tabular}

Table 1.2 shows the distance matrix for mean values of alpha power of different electrodes of all subjects in left hemisphere region. From distance matrix we observe that the value of sad mode is closer to relax mode that is less activity. It is also possible to distinguish the activity (sad , Happy) (Sad, National) and ( Sad, Romantic) and the distance is >0.2. The values of Happy, National and Romantic are little close to each other. That is natural because the National Mode and Romantic mode always corresponds to Happy Mode. The person experiences the happy emotions in National and Romantic mode. Thus it is possible to distinguish all the activities 
Table 1.3 Alpha Power EEG data of all subjects in Right Hemisphere

\begin{tabular}{|l|l|l|l|l|l|}
\hline Subject & Relax & Happy & Sad & National & Romantic \\
\hline Subject1 & 4.8856 & 3.1313 & 4.3134 & 2.8305 & 3.4134 \\
\hline Subject2 & 18.96076 & 16.83107 & 17.21783 & 18.54388 & 17.68658 \\
\hline Subject3 & 1.472763 & 0.919523 & 1.237336 & 1.224112 & 1.719605 \\
\hline Subject4 & 17.19579 & 23.20265 & 20.06566 & 19.56446 & 22.33031 \\
\hline Subject5 & 5.509194 & 2.989638 & 3.007566 & 2.84051 & 2.278586 \\
\hline Subject6 & 0.445839 & 0.558536 & 0.636776 & 0.394474 & 0.605905 \\
\hline Subject7 & 6.919211 & 1.328635 & 3.149885 & 1.075395 & 1.689013 \\
\hline Subject8 & 7.912736 & 6.994478 & 7.089778 & 6.639047 & 7.103343 \\
\hline Subject9 & 3.766678 & 1.820313 & 3.890033 & 1.655428 & 2.436842 \\
\hline Subject10 & 5.839707 & 4.407395 & 5.489906 & 4.147237 & 4.770092 \\
\hline Average & $\mathbf{7 . 2 9 0 8 2 7}$ & $\mathbf{6 . 2 1 8 3 5 4}$ & $\mathbf{6 . 6 0 9 8 1 7}$ & $\mathbf{5 . 8 9 1 5 0 4}$ & $\mathbf{6 . 4 0 3 3 6 8}$ \\
\hline
\end{tabular}

Table 1.3 shows the mean values of alpha power of different electrodes of all subjects in right hemisphere region. The table contains the mean values of alpha power of different relax mode, Happy Mode, Sad Mode, National Mode and Romantic mode. We also calculate the average values for all subjects. From table it is clear that the alpha value for relax mode is more, this means that less activity in brain for relax mode. It is also observed that we are less alert in sad mode as compared to Happy, National and Romantic mode. So it is possible to make clear distinction between the different activities in right hemisphere.

Table 1.4 Distance Matrix of Alpha Power EEG data of all subject in Right Hemisphere

\begin{tabular}{|c|l|l|l|l|l|}
\hline & \multicolumn{1}{|c|}{ Relax } & \multicolumn{1}{|c|}{ Happy } & \multicolumn{1}{|c|}{ Sad } & National & Romantic \\
\hline Relax & 0 & 1.072473 & 0.68101 & 1.399323 & 0.887459 \\
\hline Happy & 1.072473 & 0 & 0.391463 & 0.32685 & 0.185014 \\
\hline Sad & 0.68101 & 0.391463 & 0 & 0.718313 & 0.206449 \\
\hline National & 1.399323 & 0.32685 & 0.718313 & 0 & 0.511864 \\
\hline Romantic & 0.887459 & 0.185014 & 0.206449 & 0.511864 & 0 \\
\hline
\end{tabular}

The table 1.4 shows the distance matrix for mean values of alpha power of different electrodes of all subjects in left hemisphere region. From distance matrix we observe that the value of sad mode is closer to relax mode that is less activity. It is also possible to distinguish the activity (sad , Happy) (Sad, National) and ( Sad, Romantic) and the distance is $>0.2$.Also it is possible to distinguish the between ( Happy, National) (Happy, Romantic) and (National, Romantic mode).

\section{CONCLUSION}

This study successfully achieves the goal to design a system which offers offline mood recognition system. In this study we show that it is possible to recognize the different moods of person using EEG signal. The alpha powers are more alert during National, Happy, Romantic mood as compared to Sad mood. So it is possible to distinguish these different moods using alpha power values. The distance matrices also shows that it is possible to differentiate the emotions of persons using alpha power values.

\section{REFERENCES}

[1] Ito, S.-I.; Mitsukura, Y.; Fukumi, M.; Akamatsu, N.A "Feature extraction of the EEG during listening to the music using the factor analysis and neural networks." Neural Networks, Proceedings of the International Joint Conference. 2003 , Page(s): 2263 2267 vol.3 (2003)

[2] Huisheng Lu; Mingshi Wang; Hongqiang $\mathrm{Yu}$ "EEG Model and Location in Brain when Enjoying Music" Engineering in Medicine and Biology Society, 2005. IEEE-EMBS 2005. 27th Annual International Conference 2005 , Page(s): 2695 - 2698.(2005)

[3] Karthick, N.G.; Thajudin, A.V.I.; Joseph, P.K. "Music and the EEG: A Study using Nonlinear Methods" Biomedical and Pharmaceutical Engineering, 2006. ICBPE 2006. International Conference 2006 , Page(s): 424 - 427.(2006)

[4] Ito, S.-i.; Mitsukura, Y.; Fukumi, M.; Jianting Cao "Detecting method of music to match the user's mood in prefrontal cortex EEG activity using the GA". Control, Automation and Systems, 2007. ICCAS '07. International Conference .2007, Page(s): 2142 2145.(2007)

[5] Yuan-Pin Lin; Chi-Hong Wang; Tien-Lin Wu; ShyhKang Jeng; Jyh-Horng Chen "Support vector machine for EEG signal classification during listening to emotional music" Multimedia Signal Processing, 2008 IEEE 10th Workshop 2008, Page(s): 127 . 130 .(2008)

[6] Yuan-Pin Lin; Chi-Hong Wang; Tzyy-Ping Jung; Tien-Lin Wu; Shyh-Kang Jeng; Jeng-Ren Duann; Jyh-Horng Chen "EEG-Based Emotion Recognition in Music Listening" Biomedical Engineering, IEEE Transactions 2010 , Page(s): 1798 - 1806. (2010)

[7] Vijayalakshmi, K.; Sridhar, S.; Khanwani, P.” Estimation of effects of alpha music on EEGcomponents by time and frequency domain analysis" Computer and Communication Engineering (ICCCE), 2010 International Conference 2010, Page(s): 1 - 5.(2010)

[8] Janvale G.B., Gawali B. W., Deore R..S., Deshmukha S.N., Marwale A.V. and Mehrotra S.C., "Song Induced Mood Recognition System Using EEG Signals", Journal of Indian Academy of Neurosciences, ISSN 0972-7531 Volume 16, December 2009, Page No. 60. 